\title{
SUR DEUX NÉMATODES TRICHOSTRONGYLOIDES PARASITES D'UN MURIDÉ AFRICAIN
}

\author{
III. Dualité physiologique des larves infestantes, \\ liée aux rythmes saisonniers
}

\author{
M. C. DURETTE-DESSET, J. CASSONE*
}

RÉSUMÉ. La réalisation des cycles de 2 espèces de Neoheligmonella coparasites d' Uranomys ruddi à Dabou en Côte-d'Ivoire a permis de mettre en évidence que les larves infestantes (larves au stade 3) apparaissent simultanément dans les coprocultures sous deux formes différentes : engainées ou dégainées.

Les dégainées ont un pouvoir infestant beaucoup plus grand que les engainées, mais ces dernières sont susceptibles de se dégainer sous certaines conditions (" larves dégainées par vieillissement $n$ ).

Le pourcentage de dégainées au cours d'une année est plus important d'avril à octobre, période qui correspond à la saison des pluies à Dabou, dont sont originaires les Uranomys.

En tenant compte des particularités biologiques de l'hôte : sédentarité, vie solitaire, occupation de terriers toujours humides, et du fait que les Rongeurs se déparasitent rapidement, la transmission des 2 Trichostrongles est parfaitement adaptée aux conditions locales : elle semble s'effectuer selon 2 stratégies dépendant de la saison :

- Une stratégie de saison humide avec une forte apparition initiale de larves parmi lesquelles existe un nombre important de dégainées à fort pouvoir infestant.

- Une stratégie de saison sèche avec des larves engainées émises de façon plus continue, à faible pouvoir infestant, mais susceptibles de se dégainer par vieillissement et de maintenir l'infestation chez le Rongeur.

Mots-clés : Nématodes. Trichostrongyloidea. Rongeur Muridé. Larves infestantes. Zone éthiopienne.

\section{On two Trichostrongyloid Nematodes parasitic in an african Murid. III. Physiological duality of infective larvae; correlations with the seasonal rythms.}

SUMMARY. The third stage (the infective stage) of both species, Neoheligmonella dossoi and $N$. tranieri, appears simultaneously in the coprocultures in two different forms: sheathed larvae and exsheathed larvae.

The exsheathed larvae show a much higher infection rate than the sheathed ones, but the last ones could exsheathed under some conditions. These larvae are called: "larvae exsheathed by aging ".

* Laboratoire de Zoologie-Vers, associé au CNRS, Muséum National d'Histoire Naturelle, 61, rue de Buffon, F 75231 Paris Cedex 05.

Accepté le 13 aout 1987. 
The percentage of exsheathed larvae is higher from April to October, this period corresponding to the rainy season on the Ivory Coast.

Considering the host biological characteristics (sedentary, isolated, humid burrows) and the fact that the infection lasts 2 to 4 months, the transmission of the two Trichostrongylid species seems particularly well adapted to local conditions.

Transmission appears to follow two infestation strategies, depending on the season:

- A "rainy season strategy "characterized by an important initial production of larvae of which a great number are exsheathed larvae, with strong infective potential.

- A "dry season strategy " characterized by sheathed larvae, produced more regularly, having a weaker infective potential, and able to exsheathe progressively.

Key-words: Nematoda. Trichostrongyloidea. Murid Rodents. Infective larvae. Ethiopian zone.

\section{Introduction}

Dans les deux articles précédents (Durette-Desset et Cassone, 1986, 1987), nous avons décrit les adultes et les stades larvaires de deux espèces congénères Neoheligmonella dossoi et $N$. tranieri (Trichostrongyloidea) vivant dans l'intestin d'un Muridé originaire de Côte-d'Ivoire : Uranomys ruddi.

L'objet de la présente note concerne la partie libre du cycle et, plus particulièrement, la dualité physiologique du stade infestant qui apparaît sous deux formes différentes dans les coprocultures.

Cette dualité, qui semble constituer une adaptation de la biologie de ces Nématodes à la succession saison sèche-saison humide, est analysée expérimentalement.

\section{I -. Matériel et méthodes}

Le cycle des 2 espèces a été établi au Laboratoire chez l'hôte naturel en élevage. Les deux souches ont été maintenues pendant 2 ans (1984-1985).

Les premières coprocultures ont été faites à partir des œufs se trouvant dans les fèces de 12 Uranomys ruddi originaires de Dabou (Côte-d'Ivoire), rapportés à Paris en décembre 1983 par le Dr Francis Petter, du Laboratoire de Mammalogie du Muséum de Paris. Dès février 1984, les coprocultures provenaient d'animaux infestés expérimentalement.

Les fèces des Uranomys infestés naturellement ou expérimentalement sont récoltées tous les jours du Iundi au samedi inclus, les fèces émises le dimanche étant ramassées avec celles du lundi. Le poids sec moyen de ces fèces représente $0,45 \mathrm{~g}$ par Uranomys et par jour.

Les fèces sont écrasées dans un mélange en quantité approximativement égale de charbon végétal et de vermiculite (Vermex-Elfi). L'ensemble, humidifié, est déposé sur un papier filtre, dans une boîte de Pétri et mis à l'étuve à $27^{\circ}$. Les coprocultures sont humidifiées tous les jours (sauf le dimanche). 
Les coprocultures sont mises en route entre 3 semaines et un mois après l'infestation du Rongeur, ce qui correspond à la période prépatente pour les deux cycles étudiés.

Pour chaque coproculture, les larves infestantes sont récoltées une à une à la pipette dans de l'eau minérale (Volvic) et conservées à l'étuve à $27^{\circ} \mathrm{C}$, sauf dans les expérimentations où le facteur température est analysé.

Le nombre de larves récoltées résulte de deux facteurs différents qui n’ont pas été dissociés : fertilité des femelles et succès du développement des œufs jusqu'au stade 3 .

Par définition, nous appellerons " unité de coprocultures » la totalité des coprocultures effectuées à partir des fèces d'un donneur-hôte ou d'un groupe de donneurshôtes ( 2 à 4 animaux).

La durée d'une unité de coprocultures est très variable, puisqu'elle dépend d'un grand nombre de facteurs (nombre de larves données ; âge et vieillissement des larves; âge, sexe et réceptivité de l'hôte; période de l'année où l'infestation a eu lieu, self-cure de l'hôte, etc...). Cette durée varie de 3 semaines à 8 mois et est, en moyenne, de 3 mois.

D’autres méthodes de récolte ont été testées : la méthode de Baermann, classique pour récolter les larves de Trichostrongyloidea, ou la méthode de Bakarat, 1951, modifiée par Jennings, Mulligan et Urquhart, 1964 (voir Luffau, 1969). Dans le premier cas, les larves ne passent pas à travers le tamis et, dans le second, elles ne migrent pas vers le bord libre du papier.

Le pouvoir infestant des larves a été testé sur des Rongeurs infestés par voie buccale.

\section{II - Résultats obtenus}

\section{1 - Chronologie de sortie des stades 3 dans les coprocultures}

a) En infestation naturelle (expérience $42 \mathrm{KD}$ ) :

Trente et une coprocultures ont été faites du 21 décembre 1983 au 28 janvier 1984 à partir de 13 Uranomys (7 0,6 우) infestés naturellement. Les coprocultures sont vérifiées quotidiennement de J1 à J15. Les larves infestantes apparaissent à J4 (30 cas), une seule fois à J3. Des larves ont pu être dénombrées jusqu'à J13 avec un pic en J5 selon la courbe de la figure 1. Aucune larve n'a été retrouvée au-delà de $\mathrm{J} 13$.

b) En infestation expérimentale :

Quatre unités de coprocultures ont été suivies en 1984 (9 Rongeurs) et 25 en 1985 (70 Rongeurs) ${ }^{1}$.

Les larves ont été recueillies à $\mathrm{J} 4, \mathrm{~J} 10$, puis jusqu'à épuisement des copro-

1. Les chiffres journaliers concernant chaque unité de coprocultures et ayant servi aux différents calculs sont enregistrés au Laboratoire de Zoologie (Vers) sous le n MNHN 23 CU. 


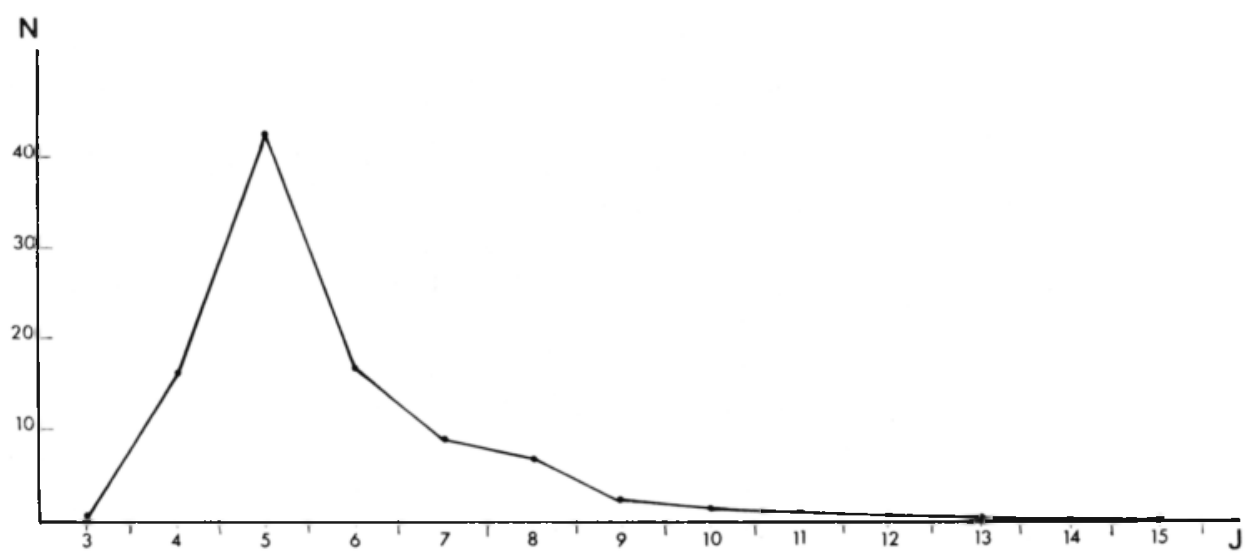

Fig. 1. - Chronologie de sortie des larves 3 dans les coprocultures (en infestation naturelle).

$\mathrm{N}=$ nombre de larves engainées et dégainées.

$\mathrm{J}=$ nombre de jours après la mise en route de la coproculture.

cultures. La présence de larves a été observée après J10 dans la moitié des coprocultures mises en route en 1984 et les trois quarts en 1985. Aucune larve n'a été retrouvée après 28 jours. Les larves dénombrées dans les coprocultures après J10 $(l)$ représentent en moyenne $10,37 \%$ de la totalité des larves récoltées en 1984 et $13,36 \%$ de celles récoltées en 1985 (T).

Le pourcentage de larves émises après 10 jours est différent selon la saison : il est plus élevé d'avril à octobre que de novembre à mars (fig. 2 , courbe en traits interrompus).

\section{2 - Dualité physiologigue du stade infestant}

Comme nous l'avons vu dans l'article précédent (Durette-Desset et Cassone, 1987), le stade 3 de $N$. dossoi est indistinguable de celui de $N$. tranieri. Mais ce stade infestant apparaît dans les coprocultures sous deux formes différentes, et ceci pour chacune des 2 espèces : soit la larve est encore enfermée dans la cuticule du $2^{\mathrm{e}}$ stade, elle est engainée; soit elle est sortie de la cuticule du $2^{\mathrm{e}}$ stade, elle est dégainée.

Différents paramètres ont été étudiés : importance de l'inoculum, âge des larves inoculées, température de la coproculture, sexe du rongeur, âge du rongeur. Aucun n'apparait avoir une influence déterminante, les 2 types de larves restent présents dans les coprocultures dans des proportions variables.

a) Analyse des histogrammes de sortie des larves par semaine dans une unité de coprocultures donnée :

Cette analyse révèle qu'il existe deux types d'histogramme :

- Dans le premier cas, l'histogramme présente des fluctuations plus ou moins régulières. Ce type se retrouve durant toute l'année (fig. 3 et 4 ). 
1984

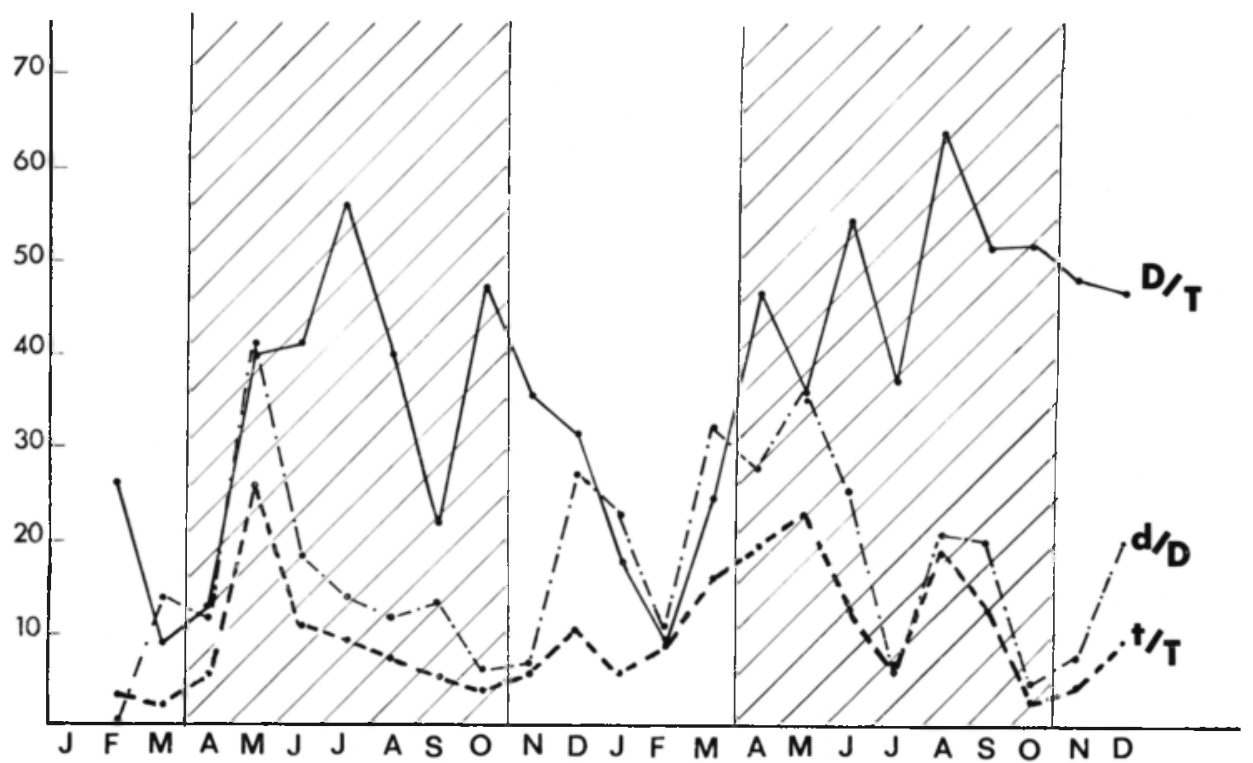

Fig. 2. - Variations du nombre de larves 3 récoltées dans les coprocultures en 1984 et 1985 , en fonction des rythmes saisonniers (en infestation expérimentale).

En abscisse, nombre de larves; en ordonnée : mois de l'année ; partie hachurée : saison humide. Courbe en traits interrompus : $t / \mathrm{T}$, pourcentage du nombre total de larves récoltées après $\mathrm{J} 10(t)$ par rapport au nombre total des larves récoltées (T).

Courbe en trait-points : $d / \mathrm{D}$, pourcentage du nombre total des larves dégainées récoltées après $\mathrm{J10}(d)$ par rapport au nombre total des larves dégainées (D).

Courbe en traits pleins : $\mathrm{D} / \mathbf{T}$, pourcentage du nombre total des larves dégainées (D) par rapport au nombre total des larves récoltées (T).

- Dans le second cas, l'histogramme débute très haut et le nombre de larves émises subit une chute brutale. Ce type d'histogramme n'a été rencontré que de la $20^{\mathrm{e}}$ semaine à la $41^{\mathrm{e}}$ semaine (fig. 5).

b) Pouvoir infestant des larves engainées et dégainées. Dégainement artificiel. Dégainement par vieillissement.

- Influence du dégainement sur le pouvoir infestant de la larve :

Les larves dégainées ont un pouvoir infestant beaucoup plus grand que les engainées. Au cours des 2 années, des rongeurs ont été infestés avec les 2 types de larves et les Vers recueillis par autopsie entre 15 jours et 3 mois après l'infestation :

- 7 rongeurs ont été infestés avec $\frac{1193}{7}$ larves engainées par individu-hôte. 1,43 Vers en moyenne ont été retrouvés (Écart-type $\sigma_{n-1}=2,06$ ). Le pourcentage de réussite est de $0,84 \%$. 

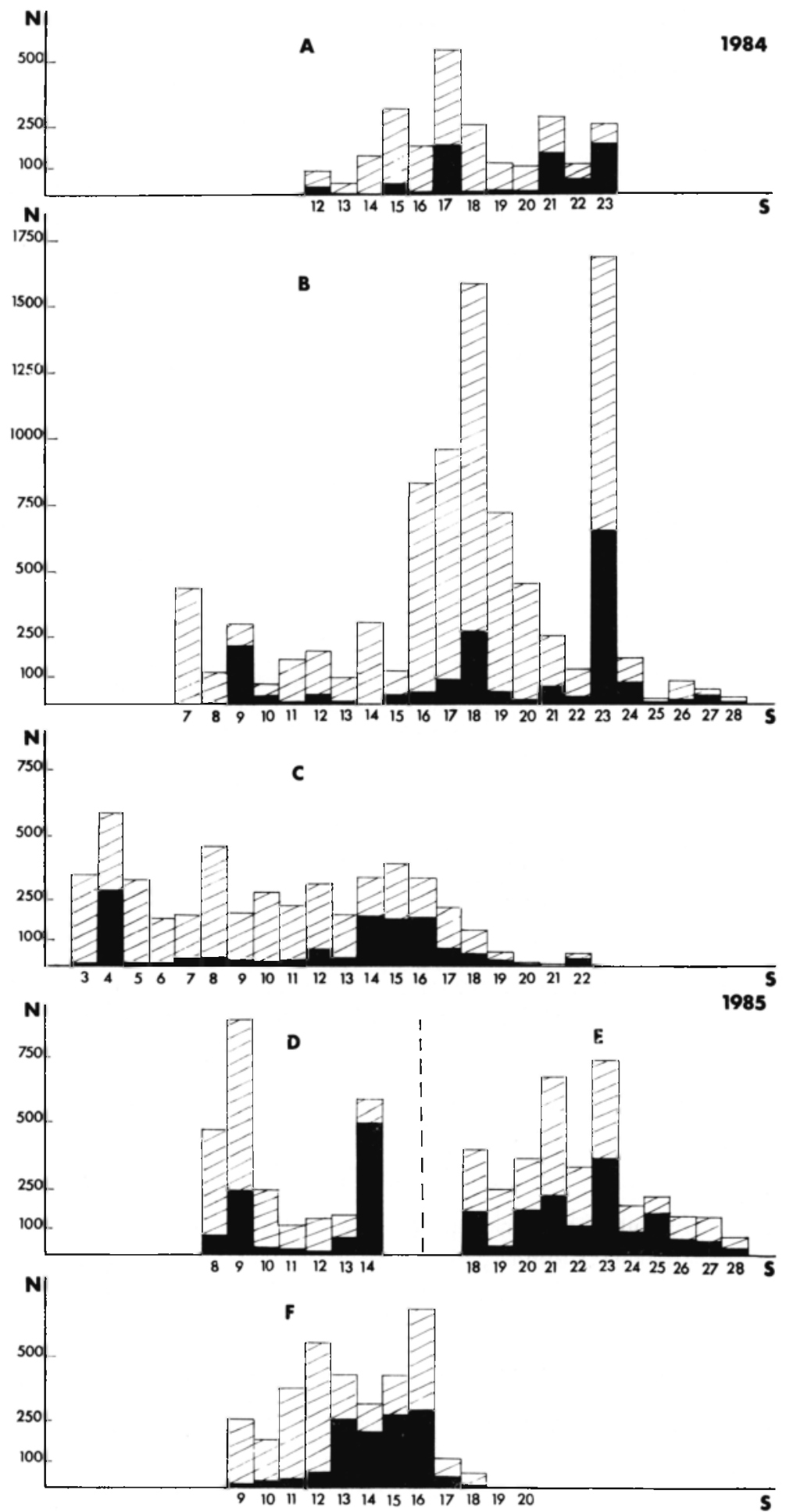

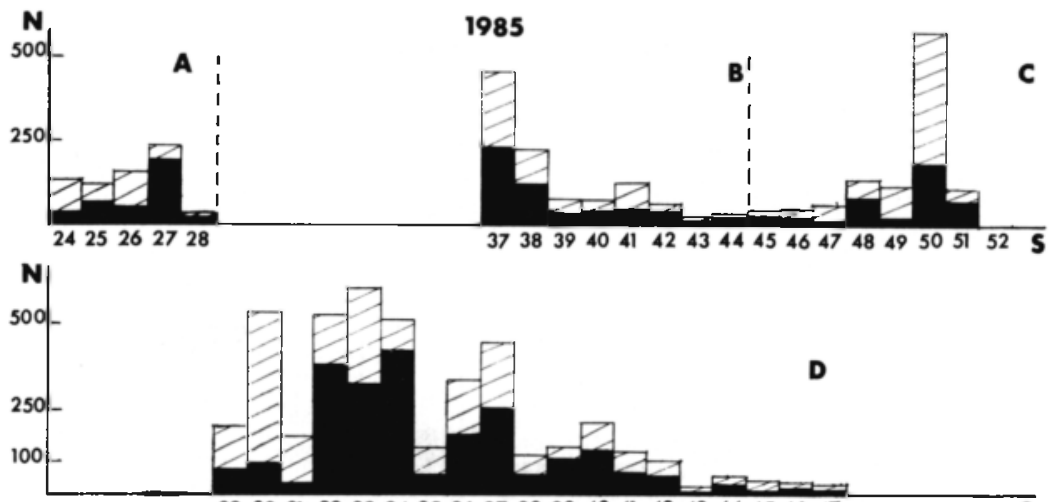

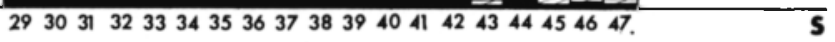

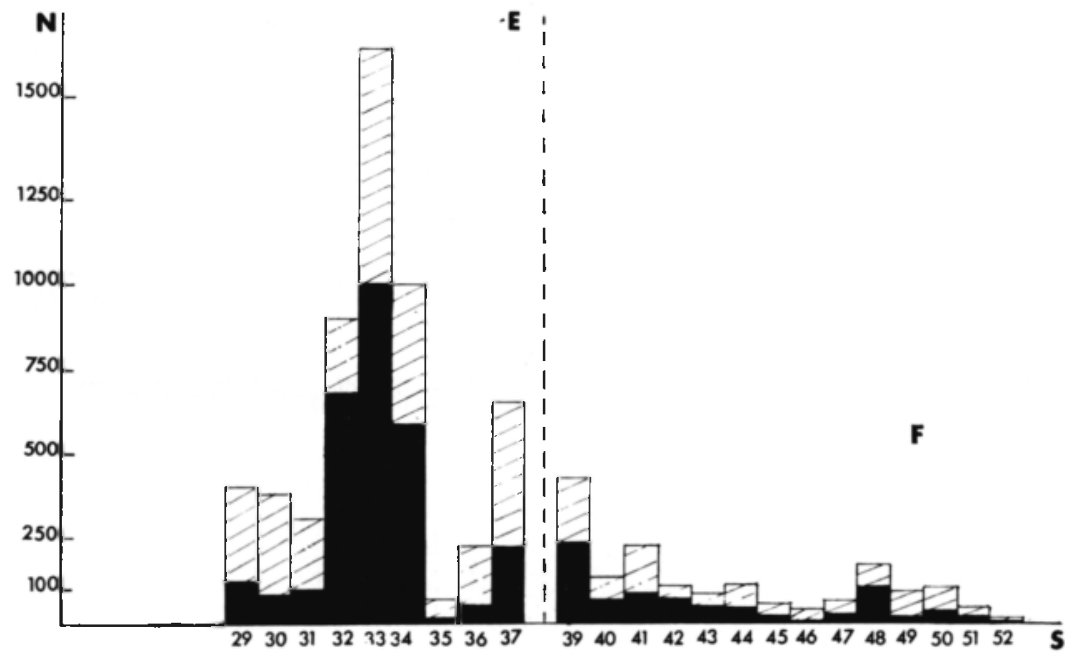

FIG. 4. - Histogramme de sortie des larves 3 dans six unités de coprocultures en 1985 (A à F) pendant les six derniers mois de l'année.

Les histogrammes présentent des fluctuations plus ou moins régulières. Ce type d'histogramme se retrouve toute l'année.

$\mathrm{N}=$ nombre total des larves recueillies; $\mathrm{S}=$ semaines de l'année; en traits pleins : nombre de larves dégainées recueillies; en traits hachurés : nombre total de larves, engainées et dégainées, recueillies.

Fig. 3. - Histogramme de sortie des larves 3 dans cinq unités de coprocultures : trois en 1984 (A, B, C) et deux en 1985 (D, E), pendant les six premiers mois de l'année.

Les histogrammes présentent des fluctuations plus ou moins régulières. Ce type d'histogramme se retrouve toute l'année.

$N=$ nombre total des larves recueillies; $S=$ semaines de l'année; en traits pleins : nombre de larves dégainées recueillies; en traits hachurés : nombre total de larves, engainées et dégainées, recueillies. 
<smiles>C=CC(C)C(C)(C)C(C)C</smiles> 
. 80 rongeurs ont été infestés avec $\frac{8858}{80}$ larves dégainées par individu-hôte. 7,02 Vers en moyenne ont été retrouvés (écart-type $\sigma_{n-1}=10,28$ ). Le pourcentage de réussite est de $6,34 \%$.

Ce pourcentage est probablement nettement inférieur à la réalité, car les animaux les plus infestés ont été conservés pour maintenir les souches.

\section{- Dégainement artificiel :}

Les larves dégainées étant plus infestantes que les engainées, nous avons tenté toute une série d'expériences pour dégainer artificiellement les larves : changements brusques de température, exposition au soleil et aux ultra-violets, assèchement suivi d'humidification à l'eau de pluie, larves mises dans de l'eau oxygénée à 10 volumes, diluée au huitième.

Tous les résultats ont été négatifs.

Par contre, nous avons obtenu le dégainement des larves en les mettant dans une solution contenant $0,03 \mathrm{ml}$ d'eau de Javel pour $20 \mathrm{ml}$ de solution de $\mathrm{NaCl}$ à $1,4 \%$ (méthode Lepage, 1934).

Les larves se dégainent entre 5 minutes et $1 / 2$ heure après avoir été mises dans la solution. 56 Rongeurs ont été infestés par cette méthode avec $\frac{7151}{56}$ larves dégainées par individu-hôte. 0,76 Vers en moyenne ont été retrouvés (écart-type $\left.\sigma_{\mathrm{n}-1}=1,54\right)$. Le pourcentage de réussite, de $0,6 \%$ est peu différent de celui obtenu en infestant avec des larves engainées.

- Dégainement par vieillissement :

Un certain nombre de larves engainées conservées à l'étuve aussi bien à $11^{\circ} \mathrm{C}$ qu'à $27^{\circ} \mathrm{C}$, se sont dégainées spontanément dans l'eau entre quelques jours à plusieurs semaines après leur apparition dans la coproculture.

Nous avons appelé de telles larves : "larves dégainées par vieillissement ".

Les expériences faites d'avril à décembre 1985 montrent que le pourcentage de dégainées par vieillissement oscille entre 7 et $21 \%$. Il n'y a pas de différence nette entre la saison sèche et la saison humide, le pourcentage moyen est d'environ $14 \%$.

Une expérience a été tentée pour tester le pouvoir infestant de telles larves. Un Rongeur a été infesté en décembre 1985 avec 47 larves dégainées par vieillisse-

FIG. 5. - Histogramme de sortie des larves 3 dans quatre unités de coprocultures en 1984.

Le nombre de larves émises initialement est très important, puis chute brutalement. Ce type d'histogramme ne se rencontre qu'entre la $20^{\mathrm{e}}$ et la $41^{\mathrm{e}}$ semaine de l'année, c'est-à-dire à une période qui correspond à la saison des pluies à Dabou. Dans l'unité de coprocultures C, l'espace blanc entre la $34^{\mathrm{e}}$ et la $38^{\mathrm{e}}$ semaine est da à l'absence de ramassage des larves durant cette période.

$\mathbf{N}=$ nombre total des larves recueillies ; $\mathbf{S}=$ semaines de l'année; en traits pleins : nombre de larves dégainées recueillies; en traits hachurés : nombre total de larves, engainées et dégainées, recueillies. 
ment après leur apparition dans la coproculture. 7 Vers ont été retrouvés, soit un pourcentage de réussite de $14,89 \%$.

Comme nous l'avons vu plus haut, environ $10 \%$ de larves 3 sont retrouvées dans les coprocultures après le $10^{\mathrm{e}}$ jour de mise en route. Parmi ces larves, le pourcentage de dégainées $(d)$ par rapport à la totalité des larves émises après J10 $(t)$ est d'environ $60 \%$ en 1984 et en 1985 . Il n'est que d'environ $40 \%$ (1984) et $50 \%$ (1985) pour les dégainées émises avant J11.

Par ailleurs, le pourcentage de dégainées émises après $\mathrm{J} 10(d)$ par rapport à la totalité des dégainées (D) est de 19,16\% en 1984 et 21,51\% en 1985 (tableau I).

\section{3 - Corrélation entre le pourGentage de larves déGainéEs et les Rythmes SAISONNIERS}

Pour chaque mois de l'année, nous avons calculé le pourcentage de larves dégainées (D) par rapport à la totalité des larves émises (T). La courbe obtenue indique que ce pourcentage est plus élevé d'avril à octobre que de novembre à mars (fig. 2, courbe en traits pleins).

Le même phénomène s'observe pour les larves émises après J10 (fig. 2, courbe en trait-point et tableau $I$ ).

Ce rythme saisonnier s'est maintenu à Paris pendant toute l'année 1984 et les trois premiers trimestres de 1985. A ce moment, le pourcentage de larves dégainées reste élevé alors qu'on arrive au mois de novembre.

Les souches ont toujours été maintenues par inoculation de larves spontanément dégainées. Il y a donc eu une sélection constante du mode de transmission par larves dégainées et il n'est pas surprenant qu'après 20 mois d'expérimentation les résultats de cette sélection se fassent sentir.

\section{TableaU I. - Variations en fonction des rythmes saisonniers du pourcentage.}

A : du nombre de larves 3 engainées et dégainées apparues dans les coprocultures après 10 jours $(t)$ par rapport au nombre total de larves recueillies (T);

B : du nombre de larves 3 dégainées apparues dans les coprocultures après 10 jours $(d)$ par rapport au nombre total de larves recueillies pendant ce laps de temps $(t)$;

$\mathrm{C}$ : du nombre de larves 3 dégainées apparues dans les coprocultures après 10 jours $(d)$ par rapport au nombre total de larves dégainées (D).

\begin{tabular}{l|c|c|c|c|c|c}
\hline & \multicolumn{2}{|c|}{$\mathrm{A}$} & \multicolumn{2}{|c|}{$\mathrm{B}$} & \multicolumn{2}{|c}{$\mathrm{c}$} \\
& \multicolumn{2}{|c|}{$\% \mathrm{t}$} & \multicolumn{2}{|c}{$\% \mathrm{~d} / \mathrm{t}$} & \multicolumn{2}{|c}{$\% \mathrm{~d} / \mathrm{D}$} \\
\cline { 2 - 7 } sur 1'année & 1984 & 1985 & 1984 & 1985 & 1984 & 1985 \\
en saison sèche & $6,4 \pm 0,3 \%$ & $13,4 \pm 0,3 \%$ & $60,9 \pm 1,6 \%$ & $67,0 \pm 1,2 \%$ & $18,0 \pm 0,7 \%$ & $21,4 \pm 0,6 \%$ \\
en saison humide & $11,5 \pm 0,3 \%$ & $15,4 \pm 0,3 \%$ & $61,0 \pm 1,6 \%$ & $71,2 \pm 1,2 \%$ & $19,2 \pm 0,7 \%$ & $21,5 \pm 0,6 \%$ \\
\hline
\end{tabular}




\section{III - Discussion}

La présence simultanée de larves infestantes engainées et dégainées dans le cycle des deux Neoheligmonella est un fait original par rapport aux autres cycles connus de Trichostrongyloidea chez lesquels la larve se présente sous l'une ou l'autre forme.

Elle est engainée chez les Trichostrongylidae, les Nematodirinae, les Ornithostrongylidae.

Chez les Molineinae, les Heligmosomidae et les Heligmonellidae, la larve se présente sous forme engainée ou dégainée. Cependant, il existe relativement peu de renseignements sur les cycles qui ne concernent pas les parasites de Ruminants, et, pour ces cycles, les auteurs ne précisent pas toujours sous quelle forme se trouve la larve infestante.

Nous n'avons trouvé que deux cas dans la littérature où des auteurs signalent la présence de larves engainées et dégainées dans le même cycle : Ow Yang, 1974, dans l'étude du cycle d'Hepatojarakus malayae Yeh, 1955, remarque que les larves engainées ou dégainées sont infestantes et ont un pouvoir infestant comparable. Mais le phénomène est très différent de ce que l'on observe chez Neoheligmonella. En fait, les larves ne se présentent pas simultanément sous forme engainée et dégainée dans les coprocultures. Suivant la méthode utilisée, l'auteur obtient soit des larves engainées (méthode de Harada et Mori, 1955), soit des larves dégainées (larves migrant sur le bord du papier-filtre).

Le second cas est celui de Nippostrongylus brasiliensis Travassos, 1914, dont le cycle a fait l'objet de très nombreux travaux et qui appartient à la même sous-famille que le genre Neoheligmonella. Pour Yokogawa, 1922, Weinstein et Jones, 1956, Haley, 1961, la larve infestante est dégainée. Pour Fahmy, 1956, Lee, 1972, elle est engainée. Enfin, pour certains auteurs, elle peut rester engainée un temps plus ou moins long (cf. Africa, 1931, Chandler, 1932, Boardman, 1933). Là encore, il semble que, lorsque la larve reste engainée, ceci est dû aux conditions dans lesquelles sont faites les coprocultures et plus particulièrement à quelle date les larves sont récoltées.

Nous avons nous-mêmes observé sur des coprocultures faites à partir des fèces de Rats infestés expérimentalement par Nippostrongylus brasiliensis que les larves infestantes étaient engainées à $\mathrm{J} 3$ après la mise en route de la coproculture et le restaient (au moins 2 mois) si on les mettait dans l'eau. Par contre, les larves récoltées au $4 \mathrm{e}$ jour étaient dégainées.

Il s'agit donc, là encore, d'un phénomène différent de celui observé chez Neoheligmonella.

Chez Neoheligmonella, la présence simultanée de larves engainées et dégainées dans la même coproculture peut s'interpréter en faisant appel aux données biologiques fournies par les hôtes. Les principales caractéristiques biologiques des 
Uranomys qui ont été étudiées par Bellier, 1968 et Gautun, 1981, sont les suivantes :

- Ils vivent dans des terriers, dans la savane herbeuse et humide.

- Ils sont sédentaires, mais peuvent se déplacer pour trouver des terriers humides même en saison sèche.

- Ils sont solitaires. Bien que les couples soient stables, ils ne vivent pas constamment ensemble. En ce qui concerne les jeunes, les femelles restent cependant plus longtemps que les mâles sur le territoire de leur mère.

Comme nous l'avons vu plus haut, ce sont les larves dégainées qui sont les plus infestantes et elles se trouvent en plus grande quantité dans les coprocultures entre avril et octobre qu'entre novembre et avril. En Côte-d'Ivoire, et plus particulièrement dans la région de Dabou d'où proviennent les Rongeurs, la saison humide s'étend précisément d'avril à octobre et la saison sèche de novembre à mars (Gautun, 1981).

Le maximum de l'infestation a donc lieu pendant la saison humide avec des larves spontanément dégainées, plus infestantes que les engainées. Mais les Rongeurs se déparasitant en moyenne entre 2 et 4 mois après le début de l'infestation ${ }^{2}$ et étant sédentaires et solitaires, l'infestation s'éteindrait rapidement s'il n'existait pas une réserve de larves.

La population de larves engainées va justement constituer cette réserve de matériel infestant qui a la possibilité de subsister puisque le terrier est constamment humide. Le pourcentage non négligeable de larves qui se dégainent et deviennent infestantes va permettre la réinfestation des Rongeurs.

\section{IV - Conclusion}

Les expériences effectuées sur les cycles de deux espèces de Neoheligmonella coparasites d'Uranomys ruddi mettent en évidence la production de deux sortes de larves :

1) Des larves engainées, plus abondantes en saison sèche (de novembre à mars). Elles sont très peu infestantes (0,84\%), mais environ $14 \%$ d'entre elles se dégainent par vieillissement et deviennent des larves dégainées, bien infestantes $(14,9 \%)$.

2) Des larves dégainées, plus abondantes en saison humide d'avril à octobre et plus infestantes (maintien des deux souches pendant deux ans).

En tenant compte des particularités biologiques de l'hôte (sédentarité, isolement, occupation de terriers humides) et du fait que les Rongeurs se déparasitent rapidement, la transmission des deux Trichostrongles paraît remarquablement bien adaptée aux conditions locales.

2. Ceci dans les conditions expérimentales. 
Elle semble s'effectuer selon deux stratégies d'infestation dépendant de la saison :

- Une stratégie de saison humide avec une forte apparition initiale de larves (fig. 5), comprenant un nombre important de dégainées à fort pouvoir infestant.

- Une stratégie de saison sèche avec des larves engainées émises de façon plus continue (figs. 3-4), à faible pouvoir infestant, susceptibles de se dégainer petit à petit et de maintenir l'infestation chez le Rongeur.

Remerciements. - Nous remercions vivement le Pr M. Lamotte, de l'École Normale Supérieure, qui nous a procuré une partie de la bibliographie, le Dr G. Luffau, de l'INRA ; Grignon, qui nous a fourni les Rats infestés par Nippostrongylus brasiliensis, et le Dr J. Cabaret, de l' INRA ; Nouzilly qui a accepté de nous conseiller sur la partie statistique de notre travail.

\section{BIBLIOGRAPHIE}

Africa C. M. : Studies on the host relations of Nippostrongylus muris with special reference to age resistance and acquired immunity. $J$. Parasitol., 1931, 18, 1-13.

Bakarat M. R. : A new procedure for the cultivation of the nematode parasites. J. Egypt. Med. Ass., 1951, 34, 323-326.

Bellier L. : Contribution à l'étude d'Uranomys ruddi Dollman. Mammalia, 1968, 32, 419-446.

BOARDMAN E. T. : A comparative study of the behaviour of the pre-parasitic larvae of four bursate nematodes. Unpublished Dissertation, Welch Medical Library, Johns Hopkins University, 1933, $121 \mathrm{pp}$.

Chandeer A. C. : Experiments on resistance of rats to superinfection with the nematode, Nippostrongylus muris. Am. J. Hyg., 1932, 16, 750-782.

Durette-Desset M.-C., Cassone J. : Sur deux Nématodes Trichostrongyloïdes. parasites d'un Muridé africain. I. Description des adultes. Ann. Parasitol. Hum. Comp., 1986, 61, 565-574.

DunetTe-Desset M.-C., CASSONE J. : Sur deux Nématodes Trichostrongylö̈des parasites d'un Muridé africain. II. Chronologie des cycles, description des stades larvaires et des immatures. Ann. Parasitol. Hum. Comp., 1987, 62, 133-158.

FAHMY M. A. : An investigation on the life cycle of Nematospiroides dubius (Nematoda: Heligmosomidae) with special reference to the free-living stages. Zeits. Parasitenkde, 1956, 17 , 394-399.

Gautur J.-C. : Écologie des Rongeurs de savane en moyenne Côte-d'Ivoire. Thèse Doctorat d'État Sci. Nat., Univ. P. et M. Curie, Paris VI, ORSTOM, Paris 1981, 1 vol. texte (pp. polycopiées) +1 vol. tableaux et graphiques.

HALEY A. J. : Biology of the rat nematode Nippostrongylus brasiliensis (Travassos, 1914). I. Systematics, hosts and geographic distribution. $J$. Parasitol., 1961, 47, 727-732.

Harada Y., Mori O. : A new method for culturing hookworm (en japonais). Yonago Acta med. I,. $1955,177-179$.

Jennings F. W., Muldigan W., Urquhart G. M. : Variables in X-Ray "Inactivation " of Nippostrongylus brasiliensis larvae. Exp. Parasitol., 1963, 13, 367-373.

LAPAGE G. : The second ecdysis of infective nematode larvae. Parasitology, 1935, 27, 186-206.

LEE D. L. : Penetration of mammalian skin by the infective larva of Nippostrongylus brasiliensis. Parasitology, 1972, 65, 499-505.

Luffau G. : Nippostrongylus brasiliensis, modèle expérimental pour l'étude de l'autostérilisation des némathelminthoses du tractus digestif. I. Cycle du parasite. Recherche vétérinaire, 1969, 3, $59-73$.

luffau G., Fougereau M., Paraf A. : Nippostrongylus brasiliensis, modèle expérimental pour l'étude de l'autostérilisation des némathelminthoses du tractus digestif. II. Modifications expérimentales du cycle évolutif. Recherche Vétérinaire, 1969, 3, 75-92.

OW-YANG C. K. : On the life history of Hepatojarakus malayae (Nematoda; Trichostrongylidae), a parasite of the feral rat in Malaysia. $J$. Helminthol., 1974, 48, 293-310.

Travassos L. : Trichostrongylideos brazileiros (III nota previa). Brazil Med., 1914, 28, 325-327.

WeINSTEIN P. P., JonES M. F. : The in vitro cultivation of Nippostrongylus muris to the adult stage. J. Parasitol., 1956, 42, 215-231.

YEH L. S. : A new bursate nematode Hepatojarakus malayae gen. et sp. nov. from the liver of Rattus rattus jarak (Bonhote) on Pulan Jarak, Straits of Malacca. J. Helminth., 1955, 29, 44-48.

Yokogawa S. : The development of Heligmosomum muris Yokogawa, a Nematode from the intestine of the mild rat. Parasitology, 1922, 14, 127-166. 